\title{
Nonsteroidal Anti-inflammatory Drugs, Apoptosis, and Colorectal Adenomas
}

\author{
CHRISTOPHER MARTIN,*, ${ }^{\ddagger}$ ALEXANDRA CONNELLY,* TEMITOPE O. KEKU, $*, \neq$ \\ SALLY B. MOUNTCASTLE, * JOSEPH GALANKO, ${ }^{\ddagger}$ JOHN T. WOOSLEY,§ BARBARA SCHLIEBE, ${ }^{\star}$ \\ P. KAY LUND,\| and ROBERT S. SANDLER*, \\ *Department of Epidemiology, School of Public Health, University of North Carolina; ${ }^{*}$ Department of Medicine and Center for Gastrointestinal \\ Biology and Disease, School of Medicine, University of North Carolina; §Department of Pathology, School of Medicine, University of North \\ Carolina; and IDepartment of Molecular Physiology, University of North Carolina, Chapel Hill, North Carolina
}

Background \& Aims: Observational studies indicate that nonsteroidal anti-inflammatory drugs (NSAIDs) reduce the risk of colorectal neoplasia. The mechanism of this effect could be via modification of apoptotic activity in colonic mucosa. We examined grossly normal rectal mucosa in patients with adenomas and adenoma-free controls to assess the associations between NSAID use, adenomatous polyps, and apoptosis. Methods: Study participants were drawn from consecutive patients who underwent colonoscopy between August, 1998, and February, 2000. Biopsy specimens were taken from normal-appearing rectal mucosa $10 \mathrm{~cm}$ from the anal verge. Apoptosis was scored from coded, H\&E-stained sections using morphologic methods. Proliferation was scored using whole crypt mitotic counts. Univariate and multivariate regression analyses were conducted to estimate crude and adjusted odds ratios (ORs). Results: There were 226 patients with adenomas and 493 adenoma-free controls. After adjusting for sex, age, race, and body mass index (BMI), individuals in the highest tertile of regular NSAID use were substantially less likely to have adenomas (OR 0.4; 95\% $\mathrm{Cl}$ : 0.2-0.7) compared with occasional or nonusers. Similarly, compared with the lowest tertile, persons in the highest tertile of rectal mucosal apoptotic activity were much less likely to have adenomas (OR 0.12; 95\% Cl: 0.07-0.20). NSAID use and apoptotic activity were not correlated $(r=0.10)$. Mucosal proliferation was not related to adenomas or NSAID use. Conclusions: Our observations suggest that NSAID use and higher levels of mucosal apoptosis are independently associated with a lower prevalence of adenomas. The study shows a strong field effect for apoptosis.

$\mathrm{O}$ $\mathrm{n}$ average, 6 people in the United States die every hour from colorectal cancer. ${ }^{1}$ Most colon cancers arise from benign, neoplastic adenomas that become severely dysplastic and develop into cancer. ${ }^{2}$ Variations in incidence of colon cancer with geography and migra- tion suggest an important environmental component to colorectal cancer risk. ${ }^{3-5}$

In recent years, nonsteroidal anti-inflammatory drugs (NSAIDs) have been investigated as a possible chemopreventive agent for colorectal cancer. The role of NSAIDs in cancer prevention was first investigated in rodent models in the early 1980s. ${ }^{6}$ NSAIDs such as aspirin, indomethacin, piroxicam, and sulindac exhibited inhibitory effects on tumors of the colon, and tumor growth often resumed when NSAIDs were discontinued. 7 The earliest case-control study investigating NSAIDs and colorectal cancer, conducted in 1988, found a $40 \%-50 \%$ reduction in colon cancer incidence among regular aspirin users. ${ }^{8}$ Subsequent epidemiologic, clinical, and experimental animal studies have found that NSAIDs decreased the risk for sporadic adenomas as well as cancer. ${ }^{9}$ Randomized trials in polyposis patients have shown a decrease in the number and size of adenomas following treatment with sulindac, a conventional NSAID, and celecoxib, a cyclooxygenase 2-specific inhibitor. ${ }^{10,11}$

The colonic mucosa experiences significant levels of spontaneous apoptosis of crypt cells. Spontaneous apoptosis may serve to remove genetically aberrant cells arising from the generally high rates of cellular proliferation in the intestinal epithelium. DNA-damaging toxins such as irradiation, chemotherapeutic agents, or toxic chemicals also induce higher rates of apoptosis, ${ }^{12}$ presumably also beneficial in removing genetically compromised cells. Thus, strategies or drugs that elevate apoptosis in the normal colonic epithelium, as well as in adenomas or cancers, may reduce adenoma or cancer risk

Abbreviations used in this paper: BMI, body mass index; NSAIDs, nonsteroidal anti-inflammatory drugs; OR, odds ratio.

(C) $\mathbf{2 0 0 2}$ by the American Gastroenterological Association 0016-5085/02/\$35.00 doi:10.1053/gast.2002.37053 
by preventing clonal expansion of genetically damaged epithelial cells.

NSAIDs may be associated with alterations in apoptosis within the gastrointestinal epithelium. ${ }^{12}$ In 1995 , Shiff et al. ${ }^{13}$ reported that sulindac and its metabolite, sulindac sulfide, could increase the rate of apoptosis of cultured HT-29 cells in a dose- and time-dependent manner. ${ }^{13}$ The same effect was found using aspirin, indomethacin, naproxen, and piroxicam. Piazza et al. ${ }^{14}$ found that sulindac sulfide and sulindac sulfone induced apoptosis in HT-29 cells. Pasricha et al. ${ }^{15}$ reported that sulindac increased apoptosis in the rectal mucosa of patients with familial polyposis and concluded that the beneficial effect on adenomas was a consequence of increased apoptosis rather than decreased proliferation.

This study investigates the interrelationships between NSAID use, apoptotic activity, and adenomatous polyps and assesses whether the protective effects of NSAIDs may arise through an apoptotic mechanism in normal mucosa.

\section{Materials and Methods Study Population}

The study was performed at the University of North Carolina Hospitals (UNCH) in Chapel Hill, North Carolina. $\mathrm{UNCH}$ is a 700-bed university hospital serving as the primary health care facility for residents of Orange and adjacent counties and as a referral center for much of the state. Patients are diverse with respect to socioeconomic status, race, and religion.

The study population was part of a large, prospective study, the Diet and Health Study III. Study participants were recruited from consecutive, consenting patients who underwent colonoscopy between August 1, 1998, and March 4, 2000, for a variety of indications or for screening. Eligible patients were 30 years of age or older; cognitively and English language proficient enough to complete a telephone risk-factor interview; and without a history of familial polyposis, colitis, previous colonic resection, previous colon cancer, or adenoma. The colonoscopy procedure had to achieve complete visualization of colon to the cecum and have had satisfactory preparation. Eligible participants were asked to allow the colonoscopist to take rectal biopsy specimens from normal mucosa and to subsequently complete a telephone interview.

The study was approved by the Committee for the Protection of the Rights of Human Subjects at the University of North Carolina School of Medicine. All participants gave written informed consent.

\section{Data Collection}

There were 2452 outpatient colonoscopies performed at the clinic during the study period. A total of 1526 subjects were ineligible for the following reasons (not mutually exclusive): previous adenomas (615), colitis (405), previous colon resection (311), under age 30 (267), previous colon cancer (220), incomplete examination (182), inability to understand consent (156), unsatisfactory preparation (149), other reasons (73), and polyposis (6). Among the 926 eligible patients, 57 $(6.2 \%)$ refused, $66(7.1 \%)$ were not asked because the research assistant was not available at the time of the procedure, and $803(93.4 \%)$ consented to participate in the study. Of the 803 consenting patients, 719 completed the NSAID usage portion of the interview, and, of these, 504 had adequate biopsy specimens for measurement of apoptosis. The indications for the procedure were abstracted from endoscopy reports using precoded forms.

Attending gastroenterologists and supervised gastroenterology fellows performed the routine colonoscopies. Six mucosal pinch biopsy specimens were obtained by the colonoscopist from consenting subjects $(\mathrm{n}=504)$. All biopsy specimens were obtained $8-10 \mathrm{~cm}$ from the anal verge using standard (8-mm wing) disposable, fenestrated colonoscopy forceps with a central spike (Wilson-Cook, Winston-Salem, NC) yielding a specimen 3-4 $\mathrm{mm}$ in diameter. Biopsy specimens were taken from normal-appearing mucosa, avoiding raised lesions or larger blood vessels. Two biopsy specimens were placed in Carnoy's fixative for later crypt dissection, and 6 biopsy specimens were placed in $10 \%$ buffered formalin for apoptosis measurement.

Apoptotic activity was scored using standard morphologic criteria applied to H\&E-stained sections viewed under light microscopy. Briefly, the paraffin-embedded biopsy specimens were sectioned into 5 sections per specimen, spaced 50 microns apart to avoid double counting of crypts. Cells were considered apoptotic if the technician observed shrinkage of the cell from its neighbors and chromatin condensation and nuclear fragmentation (karyorrhexis). Cells manifesting these criteria were included in apoptosis scores only when observed in isolated, single cells not associated with an inflammatory response. An experienced technician, blinded to adenoma status, scored all sections. We found this scoring technique to have a reproducibility of $99 \%$. Approximately 8-12 longitudinal crypts were scored per biopsy specimen, using only well-oriented crypts. We calculated the average number of apoptotic cells per crypt for each participant by combining scores from all scored crypts from that person.

Proliferation was measured using whole crypt mitotic counts on whole dissected crypts. Tissue for whole crypt dissection was transferred from Carnoy's fixative to $70 \%$ ethanol after 2.5-3.0 hours. Before staining, the tissue was rehydrated in $50 \%$ and $25 \%$ ethanol, then hydrolyzed in $1 \mathrm{~mol} / \mathrm{L}$ $\mathrm{HCl}$ at $60^{\circ} \mathrm{C}$ for 10 minutes. The biopsy specimens were then placed in Schiffs reagent and incubated in the dark for 45-60 minutes at room temperature. A small piece of the biopsy specimen was then placed on a microscope slide with $45 \%$ acetic acid and dissected under a dissecting microscope. The dissected crypts were examined under a compound microscope at $200 \times$. To be scored, the top and bottom of the crypt had to be seen. Broken crypts were not acceptable. Mitotic cells were identified by nuclear clumping, focusing through the entire 
crypt. Whole crypt mitotic count (WCMC) was determined as the average of the number of mitotic cells per crypt in 20 crypts from 2 biopsy specimens.

Lifestyle and diet questionnaires were administered to participating patients by telephone within 12 weeks of the colonoscopy. The lifestyle questionnaire included questions on characteristics such as education, age, cigarette smoking, marital status, medical history, occupation, physical activity, NSAID use, and a number of other environmental factors. The diet questionnaire was a modified Block-NCI food frequency questionnaire to ascertain usual diet over the previous 12 months. ${ }^{16}$

NSAID use was ascertained by asking participants whether, during the past 5 years, they had used any of the following medications: (1) aspirin, (2) acetaminophen, (3) pain medications or anti-inflammatory medications such as ibuprofen, Advil (Whitehall Robins, Madison, NJ), Motrin (McNeil Consumer, Fort Washington, NJ), Nuprin (Bristol Myers, New York, NY), and Alleve (Bayer Consumer, Morristown, NJ). Participants were instructed to include usage for any reason, including headaches, backaches, and/or arthritis and to include both prescription or nonprescription medicines, whether from a doctor's prescription, hospital or neighborhood clinic, pharmacy, supermarket, store, friends, neighbors, or relatives. Users were asked to categorize their usage as regular ( 3 days or more/week), occasional (less than 3 days/week), or rare (less than once/month). Regular users (3 days or more/week) were also asked for detailed information about frequency (number of uses per day or week) and duration of use (total number of weeks, months, or years each medication was taken) and, also, whether the medications were used in the past year.

The study pathologist ( J. W.) classified all polyps as adenomatous, hyperplastic, pseudopolyps, inflammatory, retention, or other. An adenoma was defined as any polyp with tubular, villoglandular, or villous pathology according to standard pathologic criteria. For analysis purposes, patients with 1 or more adenomatous polyps were classified as cases; controls were those without adenomatous polyps, although they may have had nonadenomatous polyps.

\section{Statistical Analysis}

Because the distributions of aspirin and nonaspirin NSAID use were similar and most users reported using both types, we aggregated all NSAID use for the analysis. For some analyses, NSAID use over the past 5 years was categorically defined as the following: regular (used 3 days or more/week), occasional (used less than 3 days/week), and rare (used less than $1 /$ month) or not used. For other analyses, these categories were dichotomized into regular users (used 3 days or more/week) vs. nonregular users (all others). Regular users provided detailed frequency of use data, which was used to calculate mean NSAID uses per month over the past 5 years. Tertiles of use frequency were derived from cases and controls combined for use in logistic models, with nonregular use as the reference category. Current NSAID use was defined as NSAID use during the year prior to the interview; this variable was only available for regular users.

Individual apoptotic activity scores were dichotomized at the median (2.75 apoptotic cells per crypt) for some analyses or categorized into quantiles for other analyses. Median and quantile cut points were determined using data from all participants (cases and controls).

To evaluate the association between NSAID use and the presence of colon adenomas, we used logistic regression models to estimate the odds ratio (OR) of this association while adjusting for confounders. Potential confounders were sex, age, race, body mass index (BMI), calcium, caloric intake, dietary fiber intake, percent of calorie intake from fat, family history of colon cancer, alcohol consumption, and smoking status. A covariate was considered confounding if the crude main effect estimate changed by $15 \%$ or more when the covariate was added to the crude model. Apoptosis was assessed both as a potential effect modifier and as a potential confounder of the NSAID-polyp relation. To examine the relationship between apoptosis and presence of adenomas, we used logistic analyses with the same set of potential confounders. NSAID use was evaluated in a separate model to determine whether the association between apoptosis and adenomas was confounded by NSAIDs. We also calculated nonparametric correlation statistics to assess the relationship between apoptosis and NSAIDs. All analyses were conducted using SAS, version 8.1 (SAS Institute, Cary, NC).

\section{Results}

There were 226 cases (1 or more adenomatous polyps) and 493 controls (no adenomatous polyps). Most cases $(61 \%)$ had a single adenoma, whereas $16 \%$ had 2 , $8 \%$ had $3,8 \%$ had 4 , and $7 \%$ had 5 or more adenomas. Most adenomas were found in nonrectal sites. Only $16 \%$ of cases had a rectal polyp, and, of these, $44 \%$ had multiple polyps, approximately the same ratio as cases without rectal polyps.

The demographic and lifestyle characteristics of the study population are shown in Table 1 . Cases were more likely to be men ( $53 \%$ vs. $35 \%)$ and were older than the adenoma-free controls: The mean age of cases was 60 years vs. 53 years for controls. BMI was 27.8 for all study participants and was similar between the 2 groups, 28.3 among cases vs. 27.1 among control. Most (87\%) participants had never had a colonoscopy before the study procedure.

The majority of study participants reported some NSAID use during the last 5 years. Among cases, $87 \%$ had used NSAIDs, compared with $90 \%$ of controls. Over one third of all participants were regular users (more than 3 uses/week), and regular use was more common among controls ( $45 \%$ vs. 38\%). Mean frequency of NSAID use among regular users was 22.2 uses per month. The mean 
Table 1. Characteristics of Participants by Case-Control Status

\begin{tabular}{|c|c|c|}
\hline & $\begin{array}{c}\text { Cases } \\
(\mathrm{n}=226) \\
\mathrm{n}(\%)\end{array}$ & $\begin{array}{c}\text { Controls } \\
(\mathrm{n}=493), \\
\mathrm{n}(\%)\end{array}$ \\
\hline \multicolumn{3}{|l|}{ Age $(y r)$} \\
\hline Under 45 & $14(6.2)$ & $94(19.1)$ \\
\hline $45-60$ & $102(45.1)$ & 246 (49.9) \\
\hline Over 60 & $110(48.7)$ & $153(31.0)$ \\
\hline \multicolumn{3}{|l|}{ Ethnicity } \\
\hline White & $163(72.4)$ & $376(76.7)$ \\
\hline Black & $52(23.1)$ & $101(20.6)$ \\
\hline Other & $10(4.4)$ & $15(3.1)$ \\
\hline \multicolumn{3}{|l|}{ Gender } \\
\hline Male & $120(53.1)$ & $172(34.9)$ \\
\hline Female & $106(46.9)$ & $320(64.9)$ \\
\hline \multicolumn{3}{|l|}{ Education } \\
\hline Less than high school & $56(24.8)$ & $88(18.0)$ \\
\hline High school graduate & $116(51.3)$ & $295(60.2)$ \\
\hline Some college & $54(23.9)$ & $109(22.2)$ \\
\hline \multicolumn{3}{|l|}{ Marital status } \\
\hline Not married & $71(31.4)$ & $163(33.1)$ \\
\hline Married & $155(68.6)$ & $329(66.7)$ \\
\hline \multicolumn{3}{|l|}{ Previous colonoscopy } \\
\hline Yes & $27(12.0)$ & $70(14.2)$ \\
\hline No & $199(88.0)$ & $423(85.8)$ \\
\hline \multicolumn{3}{|c|}{ History of cancer (not colorectal) } \\
\hline Yes & $28(12.9)$ & $57(11.5)$ \\
\hline No & $196(86.7)$ & $431(87.4)$ \\
\hline Don’t know & $2(0.9)$ & $5(1.1)$ \\
\hline \multicolumn{3}{|l|}{ Ever smoked $>100$ cigarettes } \\
\hline Yes & $125(55.3)$ & $247(50.1)$ \\
\hline No & $101(44.7)$ & 246 (49.9) \\
\hline \multicolumn{3}{|l|}{ Current Smoking } \\
\hline Current smoker & $35(15.5)$ & $95(19.3)$ \\
\hline Ex-smoker & $88(38.9)$ & $149(30.22)$ \\
\hline Never smoked & $101(44.7)$ & 246 (49.9) \\
\hline \multicolumn{3}{|l|}{ Body mass index (kg/m²) } \\
\hline Underweight $(<18.5)$ & $5(2.2)$ & $12(2.4)$ \\
\hline Normal (18.5-24.9) & $70(31.0)$ & $182(36.9)$ \\
\hline Overweight (25.0-29.9) & 69 (30.5) & $158(32.1)$ \\
\hline Obese (30.0-39.9) & $68(30.1)$ & $114(23.1)$ \\
\hline Extremely obese $(\geq 40.0)$ & $14(6.2)$ & $27(5.5)$ \\
\hline \multicolumn{3}{|c|}{$\begin{array}{l}\text { Mean NSAID use/month in past } \\
5 \mathrm{yr}\end{array}$} \\
\hline 1st tertile & $30(13.6)$ & $71(14.7)$ \\
\hline 2nd tertile & $24(10.9)$ & $72(14.9)$ \\
\hline 3rd tertile & $31(14.0)$ & $78(16.1)$ \\
\hline Occasional/rare/no use & $136(60.2)$ & $263(53.6)$ \\
\hline \multicolumn{3}{|l|}{ Indications $^{a}$} \\
\hline Pain & $24(11)$ & $82(17)$ \\
\hline Constipation & $14(6)$ & $37(8)$ \\
\hline Diarrhea & $10(4)$ & $52(11)$ \\
\hline Family history & $51(23)$ & $143(29)$ \\
\hline Bleeding $b$ & $114(50)$ & 269 (55) \\
\hline X-ray abnormality & $6(3)$ & $6(3)$ \\
\hline Other & $45(20)$ & $135(27)$ \\
\hline
\end{tabular}

NOTE. Missing data: ethnicity (1), gender (1), education (1), current smoking (5), NSAID use (12).

${ }^{a}$ Categories are not mutually exclusive.

${ }^{b}$ Bleeding includes melena, hematochezia, positive fecal occult blood test, and iron deficiency anemia. uses per month within tertiles of use frequency was 3.4 for the first tertile, 14.3 for the second tertile, and 47.3 for the third tertile. Almost all regular users reported NSAID use during the past year.

All of the participants in the study were referred for colonoscopy. The indication for the procedure is shown in Table 1 . These indications are not mutually exclusive. The most common indication for colonoscopy in this population was bleeding (hematochezia, melena, positive fecal occult blood test, and iron deficiency anemia). The proportion of cases with this indication $(50 \%)$ was virtually the same as that for controls (55\%). Each of the indications was similar in cases and controls.

\section{NSAID Use and Adenomas}

Results of logistic regression modeling of the relation between frequency of NSAID use and colorectal adenomas are presented in Table 2. All ORs in Table 2 are adjusted for age, sex, BMI, and race and are based on comparisons with a common reference group of nonregular users (used less than 3 times/month). The effect of NSAIDs was strongest in the most frequent users. Persons in the highest tertile of regular NSAID use were least likely to have an adenoma (OR 0.5; 95\% CI: $0.3-0.8)$.

\section{Proliferation, Apoptosis, and Adenomas}

The proliferation index for patients with adenomas ranged from 2.8 to 9.4 with mean (SE) equal to 4.83 (1.08), similar to the range in controls (2.9-10.2), with a mean (SE) of 4.87 (1.22). The proliferation index was not different between the patients with adenomas and those without ( $P=0.17$ for difference in means). There was no correlation between proliferation and NSAID use (Pearson's $r=0.04$ ).

The median apoptosis score (mean number of apoptotic cells per crypt) for the entire study population was 2.75 apoptotic cells/crypt. Apoptosis scores differed significantly between adenoma cases (mean $=2.46$ ) and

Table 2. Frequency of NSAID Use and Presence of Adenomas

\begin{tabular}{|c|c|c|}
\hline NSAID use frequency over past 5 years $^{a}$ & $\mathrm{n}$ & $\begin{array}{l}\text { Odds ratio } \\
(95 \% \mathrm{Cl})^{b}\end{array}$ \\
\hline Nonregular use ${ }^{c}$ & 400 & 1.0 (reference) \\
\hline \multicolumn{3}{|l|}{ Regular use $^{d}$ (mean use per month; range) } \\
\hline Tertile $1(3.4 ; 0.1-6.96)$ & 101 & $0.83(0.5-1.4)$ \\
\hline Tertile 2 (14.3; 7.8-23.5) & 96 & $0.51(0.3-0.9)$ \\
\hline Tertile 3 (47.3; 24.4-365.3) & 109 & $0.50(0.3-0.8)$ \\
\hline
\end{tabular}

${ }^{a}$ Mean uses of NSAIDs per month over previous 60 months.

${ }^{b}$ Adjusted for age, sex, body mass index (BMI), and race.

'Less than 3 uses per week; includes occasional, rare, and non use. ${ }^{d}$ At least 3 uses/week. 
Table 3. Mean Apoptosis Scores by Participant Characteristics

\begin{tabular}{|c|c|c|}
\hline & $\begin{array}{l}\text { Mean apoptosis } \\
\text { score }\end{array}$ & $P$ value \\
\hline Overall & 2.77 & \\
\hline Case status & & $<0.0001$ \\
\hline Adenoma & 2.46 & \\
\hline No adenomas & 2.94 & \\
\hline Age $(y r)$ & & 0.3768 \\
\hline Under 45 & 2.87 & \\
\hline $45-60$ & 2.76 & \\
\hline Over 60 & 2.75 & \\
\hline Ethnicity & & 0.912 \\
\hline White & 2.79 & \\
\hline Black & 2.76 & \\
\hline Other & 2.79 & \\
\hline Sex & & 0.065 \\
\hline Male & 2.70 & \\
\hline Female & 2.82 & \\
\hline Current smoking status & & 0.189 \\
\hline Current smoker & 2.86 & \\
\hline Ex-smoker & 2.70 & \\
\hline Never smoked & 2.79 & \\
\hline Total calcium intake & & 0.223 \\
\hline Low & 2.70 & \\
\hline Moderate & 2.82 & \\
\hline High & 2.80 & \\
\hline Percentage calories from fat & & 0.020 \\
\hline$<30 \%$ & 2.90 & \\
\hline $30 \%-40 \%$ & 2.72 & \\
\hline$>40 \%$ & 2.71 & \\
\hline Body mass index $\left(\mathrm{kb} / \mathrm{m}^{2}\right)$ & & 0.149 \\
\hline Underweight $(<18.5)$ & 3.11 & \\
\hline Normal (18.5-24.9) & 2.82 & \\
\hline Overweight (25.0-29.9) & 2.76 & \\
\hline Obese $(>29.9)$ & 2.71 & \\
\hline Total fiber intake & & 0.131 \\
\hline Low & 2.72 & \\
\hline Moderate & 2.72 & \\
\hline High & 2.85 & \\
\hline Use of tofu or meat substitutes & & 0.991 \\
\hline Yes & 2.77 & \\
\hline No & 2.77 & \\
\hline
\end{tabular}

controls (mean $=2.94)$ when compared in an analysis of variance procedure $(\mathrm{F}<0.0001)$. Nearly twice as many $(77 \%)$ cases as controls $(36 \%)$ were at or below the population median of 2.75 cells/crypt.

The distribution of apoptosis scores is shown in Table 3 with respect to a number of dietary and lifestyle factors. Apoptotic activity was higher in underweight and normal BMI participants and among those whose energy intake from fat was less than $30 \%$ of total caloric intake. The amount of dietary fiber consumption was positively associated with apoptosis. Despite reports of laboratory evidence of induction of apoptosis in colonic cancer cells by plant flavones, ${ }^{17}$ apoptosis scores in our study population did not differ between persons reporting tofu consumption and nontofu consumers. Apoptotic activity decreased somewhat with age. Mean apoptosis scores did not differ between cases having rectal vs. nonrectal polyps (data not shown).

The relationship of apoptosis to the presence of adenomas was assessed in logistic regression models using tertiles of apoptosis scores; these results are shown in Table 4. Based on our a priori change-in-estimate criterion, none of the covariates appeared to confound the apoptosis-adenoma relationship; thus the ORs presented are unadjusted. Overall, persons with higher apoptotic activity were much less likely to have colorectal adenomas. Compared with persons in the lowest tertile of apoptosis scores, adenomatous polyps were nearly 10 times less prevalent among those in the highest tertile (OR 0.12; 95\% CI: $0.07-0.20$ ) and 60\% less prevalent among the middle tertile of apoptosis (OR 0.40; $95 \%$ CI: 0.26-0.63). These results did not change after excluding cases with rectal adenomas (data not shown).

\section{NSAID and Apoptosis}

There was little evidence of a relationship between NSAID use and apoptotic activity. Among all participants, the Spearman correlation coefficient between apoptotic activity and NSAID use per month was $<0.10$.

Because NSAIDs and apoptosis each predict adenoma status, an observed relationship between NSAIDs and apoptosis in the entire study population might reflect unmeasured adenoma causal factors independently correlated with these exposures. We therefore restricted further analyses of the NSAIDs-apoptosis relationship to adenoma-free controls only (Table 5). Mean apoptosis scores were 2.86, 2.92, and 2.99 among regular NSAID users, occasional users, and rare or nonusers, respectively; however, these scores did not differ statistically for any of

Table 4. Association Between Apoptosis Scores (Tertiles) and Presence of Adenomas

\begin{tabular}{|c|c|c|c|c|c|}
\hline Apoptosis tertile & $\begin{array}{c}\text { Range of } \\
\text { apoptosis scores }\end{array}$ & $\begin{array}{c}\text { Cases } \\
(n=174)\end{array}$ & $\begin{array}{l}\text { Controls } \\
(n=330)\end{array}$ & $\begin{array}{c}\text { Mean apoptosis } \\
\text { score }\end{array}$ & Odds ratio $(95 \% \mathrm{Cl})$ \\
\hline $1^{a}$ & $1.29-2.44$ & 94 & 73 & 2.06 & 1.00 \\
\hline 2 & $2.46-3.02$ & 58 & 110 & 2.74 & $0.40(0.26-0.63)$ \\
\hline 3 & $3.02-6.92$ & 22 & 147 & 3.52 & $0.12(0.07-0.20)$ \\
\hline
\end{tabular}

aReference category. 
Table 5. Apoptotic Scores and Distribution of Apoptotic Activity Among Controls by NSAID Use Category

\begin{tabular}{lcccc}
\hline & \multicolumn{3}{c}{ NSAID use category, mean (SD) } \\
\cline { 2 - 4 } & $\begin{array}{c}\text { Never used } \\
(\mathrm{n}=34)\end{array}$ & $\begin{array}{c}\text { Occasional users } \\
(\mathrm{n}=141)\end{array}$ & $\begin{array}{c}\text { Regular users } \\
(\mathrm{n}=154)\end{array}$ & Bonferroni test \\
\hline $\begin{array}{l}2.86(0.51) \\
\mathrm{n}(\text { row\%) }\end{array}$ & $\begin{array}{c}2.92(0.70) \\
\mathrm{n}(\text { row\%) }\end{array}$ & $2.99(0.75)$ & $\mathrm{F}=0.65(P=0.5218)$ \\
Apoptosis score tertile ${ }^{a}$ & $7(20.6)$ & $33(23.4)$ & $32(20.8)$ & Mantel-Haenszel $\chi^{2}=1.51$, \\
1 & $13(38.2)$ & $51(36.2)$ & $46(29.9)$ & 1 df $(P=0.2194)$ \\
2 & $14(41.2)$ & $57(40.4)$ & $76(49.4)$ & \\
3 & & & & \\
\hline
\end{tabular}

${ }^{a}$ Cut points derived from cases and controls.

the pairwise comparisons (Bonferroni statistic $=0.52$ ). In addition, using cut points derived from cases and controls, the proportion of controls in each tertile of apoptosis scores did not differ within strata defined by frequency of NSAID use $\left(\chi^{2}=1.51, P=0.22\right)$.

To assess whether the protective effect of NSAIDs might be mediated by apoptosis, we compared the magnitude of the NSAID-adenoma association within strata defined by the median value for apoptosis scores. The association was similar in both strata. Similarly, there was no difference in the strength of the apoptosis-adenoma relationship within strata defined by NSAID use. When we added apoptosis scores to a logistic model of NSAID use and adenomas, the OR for NSAID use was unchanged, providing no evidence for confounding of the NSAID-adenoma relationship by apoptosis. Neither was NSAID use confounding the association between apoptosis and adenomas because the apoptosis-adenoma OR did not change when NSAID use was added to that model.

\section{Discussion}

Adenomas are the precursors to the majority of large bowel malignancies. Previously, there has been a widespread belief that adenomas arise from mucosa with higher rates of proliferation, although it has been more recently recognized that mucosal homeostasis requires a balance between proliferation and apoptosis. ${ }^{18}$ In the present study, we measured both proliferation and apoptosis in normal rectal mucosa. Although we found no association between rectal mucosal proliferation and adenomas, we observed a substantial association between lower apoptotic activity and adenomas elsewhere in the large bowel, thus providing support for the elusive "field effect." The study also confirmed the protective effect of NSAID, but, contrary to expectation, there was no evidence that the NSAID effect was mediated through apoptosis.

The association between proliferation in the normal mucosa and adenoma risk has been difficult to prove in clinic-based studies of average risk individuals. ${ }^{19}$ In a prospective study of participants in a polyp chemoprevention study, there was no association between proliferation measured by proliferating cell nuclear antigen (PCNA) immunohistochemistry and current or future adenoma risk. ${ }^{20}$ In a previous study using a population similar to the present study, we found no relationship between proliferation measured by PCNA immunohistochemistry or whole crypt mitotic counts and adenoma risk. ${ }^{21}$ In the current study, we measured proliferation by counting mitotic figures in microdissected crypts. Scoring mitoses in microdissected crypts is thought, by some, to be the method of choice for the study of gastrointestinal proliferation. ${ }^{22}$ The technique has been validated in human intestinal biopsy samples and animal studies. ${ }^{23,24}$ Using this technique, we again found no association between proliferation and adenoma risk.

In contrast, the present study demonstrated a very strong inverse association between adenomas and apoptosis measured in biopsy specimens taken from normal mucosa. The biopsy specimens were not from the adenomatous tissues or from mucosa in the vicinity of adenomas. The observation of an association between apoptosis in the rectum and adenomas elsewhere in the colon is consistent with a "field effect." Given the dose-response relationship and the magnitude of the effect (participants in the highest tertile of apoptosis were almost 90\% less likely to have an adenoma), this finding is not likely due to chance. The study provides convincing evidence that patients with adenomas have a generalized decreased level of apoptosis throughout their colons. One might speculate that this low level of apoptosis is responsible for the development of adenomas. The factors that are responsible for variable levels of apoptosis in normal mucosa are not known. In the present study, we found an inverse association with dietary fat, a known risk factor for colorectal neoplasia.

The present study also showed a strong protective effect of NSAIDs on adenomas. Individuals in the high- 
est category of NSAID use were about half as likely to have adenomas as nonusers. These findings are consistent with a previous study in a similar population ${ }^{9}$ and with a large body of evidence from epidemiologic studies. ${ }^{25}$

Several mechanisms have been proposed to explain the possible protective mechanism against colorectal neoplasia exhibited by nonsteroidal, anti-inflammatory agents. NSAID may reduce risk through their inhibitory effects on prostaglandins. Experimental animal and human tumors have been shown to contain or produce large quantities of prostaglandins, ${ }^{26,27}$ and prostaglandin synthesis has been shown to be associated with tumor promotion and metastatic potential. ${ }^{28}$ Another hypothesized mechanism by which NSAIDs may exert their antineoplastic effects in the gastrointestinal tract is via induction of apoptosis.

The results of our study, however, suggest that the protective effect of NSAIDs in average-risk individuals is independent of apoptotic activity in normal mucosa. These results are seemingly at odds with previous research. Prior studies have shown, for example, that both conventional NSAIDs and selective cyclooxygenase 2 inhibitors can lead to the regression of colorectal adenomas in patients with familial adenomatous polyposis coli (FAP). ${ }^{10,11}$ Apoptosis has been the presumed mechanism for this regression. To explain the apparent discrepancy in our findings, we would postulate that the effect of NSAIDs on apoptosis is limited to the adenomas themselves as suggested by Ahnen. ${ }^{29}$ We hypothesize that the protective effect of NSAIDs is a consequence of their exclusive effect on adenomatous tissue in which cyclooxygenase 2 is up-regulated and not on normal mucosa. Support for this hypothesis comes from a recent study of young phenotypically negative FAP patients. ${ }^{30}$ In that study, sulindac did not affect apoptosis in biopsy specimens from normal-appearing rectal mucosa, suggesting that the effect of NSAIDs is limited to transitional mucosa in which cell kinetics are disturbed. The challenge for the future is to discover the factors that regulate apoptosis in the nonadenomatous mucosa.

This study provides several advantages over previously published studies. The study was designed to simultaneously measure proliferation, apoptosis, NSAID use, and adenomas in a population of average risk subjects undergoing complete colonoscopy to the cecum. The study, therefore, permitted examination of the effect of certain environmental exposures, such as NSAIDs, on the normal rectal mucosa. We obtained very detailed information concerning the frequency and duration of NSAID use. The biopsy specimens were uniformly obtained from the same rectal site, and the same pathologist reviewed all pathology slides using predefined criteria. Participants provided comprehensive information concerning potentially confounding dietary and lifestyle factors.

The study had certain limitations. NSAID use was based on self-report, and self-report is susceptible to error. Although the study had an excellent response rate $(93 \%)$ overall, there were 215 subjects who chose not to participate in the biopsy portion of the study. Whether nonparticipation in this portion of the study introduced bias is not known, although it is unlikely that consenting to a biopsy is related to apoptotic activity. Finally, both apoptosis and proliferation were measured by morphologic methods. Although these techniques are currently the standard, they may not be a complete measure of these activities.

In conclusion, the present study has confirmed the strong negative association between NSAIDs and colorectal adenomas. Individuals in the highest tertile of regular NSAID consumption were $50 \%$ less likely to have an adenoma as occasional or nonusers. There was also a striking negative association between apoptosis measured in normal-appearing rectal mucosa and adenomas found elsewhere in the colon. Participants in the highest category of apoptosis were almost $90 \%$ less likely to have an adenoma. The apoptosis results are consistent with the existence of a field effect in the large bowel that predisposes individuals with lower levels of apoptosis to the development of adenomas. Finally, the results of the present study suggest that the protective effect of NSAIDs on adenomas is not mediated through apoptosis in the normal mucosa. The factors that are responsible for varying levels of apoptosis in the normal rectal mucosa deserve further study because they may provide opportunities for prevention of colorectal neoplasia.

\section{References}

1. Hamilton SR. Origin of colorectal cancers in hyperplastic polyps and serrated adenomas: another truism bites the dust. J Natl Cancer Inst 2001;93:1282-1283.

2. Moss SF, Liu TC, Petrotos A, Hsu TM, Gold LI, Holt PR. Inward growth of colonic adenomatous polyps. Gastroenterology 1996; 111:1425-1432.

3. Haenszel W, Kurihara M. Studies of Japanese migrants. I. Mortality from cancer and other diseases among Japanese in the United States. J Natl Cancer Inst 1968;40:6843-6868.

4. Whittemore AS. Colorectal cancer incidence among Chinese in North America and the People's Republic of China: variation with sex, age, and anatomical site. Int J Epidemiol 1989;18:563568.

5. Thomas DB, Karagas MR. Cancer in first and second generation Americans. Cancer Res 1987;47:5771-5776.

6. Pollard M, Luckert PH. Indomethacin treatment of rats with dimethylhydrazine-induced intestinal tumors. Cancer Treat Rep 1980;64:1323-1327.

7. Reddy BS, Rao CV, Rivenson A, Kelloff G. Inhibitory effect of 
aspirin on azoxymethane-induced colon carcinogenesis in F344 rats. Carcinogenesis 1993;14:1493-1497.

8. Kune GA, Kune S, Watson LF. Colorectal cancer risk, chronic illnesses, operations, and medications: case control results from the Melbourne Colorectal Cancer Study. Cancer Res 1988;48: 4399-4404.

9. Sandler RS, Galanko JC, Murray SC, Helm JF, Woosley JT. Aspirin and nonsteroidal anti-inflammatory agents and risk for colorectal adenomas. Gastroenterology 1998;114:441-447.

10. Giardiello FM, Hamilton SR, Krush AJ, Piantadosi S, Hylind LM, Celano P, Booker SV, Robinson CR, Offerhaus GJ. Treatment of colonic and rectal adenomas with sulindac in familial adenomatous polyposis. N Engl J Med 1993;328:1313-1316.

11. Steinbach G, Lynch PM, Phillips RK, Wallace MH, Hawk E, Gordon GB, Wakabayashi N, Saunders B, Shen Y, Fujimura T, Su LK, Levin B. The effect of celecoxib, a cyclooxygenase-2 inhibitor, in familial adenomatous polyposis. N Engl J Med 2000;342:19461952.

12. Wong BC, Zhu GH, Lam SK. Aspirin induced apoptosis in gastric cancer cells. Biomed Pharmacother 1999;53:315-318.

13. Shiff SJ, Qiao L, Tsai LL, Rigas B. Sulindac sulfide, an aspirin-like compound, inhibits proliferation, causes cell cycle quiescence, and induces apoptosis in HT-29 colon adenocarcinoma cells. J Clin Invest 1995;96:491-503.

14. Piazza GA, Rahm AL, Krutzsch M, Sperl G, Paranka NS, Gross PH, Brendel K, Burt RW, Alberts DS, Pamukcu R. Antineoplastic drugs sulindac sulfide and sulfone inhibit cell growth by inducing apoptosis. Cancer Res 1995;55:3110-3116.

15. Pasricha PJ, Bedi A, O'Connor K, Rashid A, Akhtar AJ, Zahurak ML, Piantadosi S, Hamilton SR, Giardiello FM. The effects of sulindac on colorectal proliferation and apoptosis in familial adenomatous polyposis. Gastroenterology 1995;109:994-998.

16. Block G, Hartman AM, Dresser CM, Carroll MD, Gannon J, Gardner L. A data-based approach to diet questionnaire design and testing. Am J Epidemiol 1986;124:453-469.

17. Wenzel U, Kuntz S, Brendel MD, Daniel H. Dietary flavone is a potent apoptosis inducer in human colon carcinoma cells. Cancer Res 2000;60:3823-3831.

18. Bedi A, Pasricha PJ, Akhtar AJ, Barber JP, Bedi GC, Giardiello FM, Zehnbauer BA, Hamilton SR, Jones RJ. Inhibition of apoptosis during development of colorectal cancer. Cancer Res 1995;55: 1811-1816.

19. Ahnen DJ, Byers T. Proliferation happens. JAMA 1998;280: 1095-1096.

20. Sandler RS, Baron JA, Tosteson TD, Mandel JS, Haile RW. Rectal mucosal proliferation and risk of colorectal adenomas: results from a randomized controlled trial. Cancer Epidemiol Biomarkers Prev 2000;9:653-656.

21. Keku TO, Galanko JA, Murray SC, Woosley JT, Sandler RS. Rectal mucosal proliferation, dietary factors, and the risk of colorectal adenomas. Cancer Epidemiol Biomarkers Prev 1998;7:993999.

22. Wong WM, Mandir N, Goodlad RA, Wong BC, Garcia SB, Lam SK, Wright NA. Histogenesis of human colorectal adenomas and hyperplastic polyps: the role of cell proliferation and crypt fission. Gut 2002;50:212-217.

23. Mills SJ, Mathers JC, Chapman PD, Burn J, Gunn A. Colonic crypt cell proliferation state assessed by whole crypt microdissection in sporadic neoplasia and familial adenomatous polyposis. Gut 2001;48:41-46.

24. Goodlad RA, Levi S, Lee CY, Mandir N, Hodgson H, Wright NA. Morphometry and cell proliferation in endoscopic biopsies: evaluation of a technique. Gastroenterology 1991;101:1235-1241.

25. Sandler RS. Aspirin and other nonsteroidal anti-inflammatory agents in the prevention of colorectal cancer. Important Adv Oncol 1996;123-137.

26. Rigas B, Goldman IS, Levine L. Altered eicosanoid levels in human colon cancer. J Lab Clin Med 1993;122:518-523.

27. Suh O, Mettlin C, Petrelli NJ. Aspirin use, cancer, and polyps of the large bowel. Cancer 1993;72:1171-1177.

28. Hixson LJ, Alberts DS, Krutzsch M, Einsphar J, Brendel K, Gross $\mathrm{PH}$, Paranka NS, Baier M, Emerson S, Pamukcu R. Antiproliferative effect of nonsteroidal anti-inflammatory drugs against human colon cancer cells. Cancer Epidemiol Biomarkers Prev 1994;3: 433-438.

29. Ahnen DJ. Colon cancer prevention by NSAIDs: what is the mechanism of action? Eur J Surg 1998;(Suppl)111-114.

30. Keller JJ, Johan G, Offerhaus A, Hylind LM, Giardiello FM. Rectal epithelial apoptosis does not predict response to sulindac treatment or polyp development in presymptomatic familial adenomatous polyposis patients. Cancer Epidemiol Biomarkers Prev 2002;670-671.

Received March 13, 2002. Accepted August 23, 2002.

Address requests for reprints to: Robert S. Sandler, M.D., M.P.H., Division of Digestive Diseases and Nutrition, CB 7080, 719 BurnettWomack Building, University of North Carolina, Chapel Hill, North Carolina 27599-7080. e-mail: rsandler@med.unc.edu; fax: 919-9666842.

Supported in part by grants from the National Institutes of Health (P30 DK34987, T32 DK07634, and R01 CA 44684). 